\title{
Impact of increased aspartate aminotransferase to alanine aminotransferase (De Ritis) ratio in prognosis of testicular cancer
}

\author{
Sacit Nuri Gorgel ${ }^{1, *}$, Yigit Akin ${ }^{1, *}$, Esra Meltem Koc ${ }^{2}$, Osman Kose', Serkan Ozcan ${ }^{1}$, Yuksel Yilmaz ${ }^{1}$ \\ Departments of 'Urology and ${ }^{2}$ Family Medicine, Izmir Katip Celebi University School of Medicine, Izmir, Turkey
}

Purpose: Imaging studies can show metastasis in testicular cancer (TCa); however, a test for risk of metastasis in TCa has not been described. The ratio of aspartate aminotransferase to alanine aminotransferase, also called the De Ritis ratio (DRR), is used for many other malignancies. We aimed to evaluate the association between preoperatively assessed DRR and prognosis in patients with TCa.

Materials and Methods: One hundred twenty-eight patients with TCa were enrolled in a retrospective study between March 2007 and January 2017. Clinical, biochemical, and pathological data were recorded. Univariate and multivariate logistic regression analyses were used. The prognostic value of DRR and the threshold value were assessed by use of receiver operating characteristic curves. Significance was defined as $p<0.05$.

Results: Mean follow-up was 37 \pm 9.7 months. There were 45 and 73 TCa patients with and without lymph node metastasis, respectively. Lung metastases and other solid organ metastases occurred in 14 and 4 patients, respectively. The optimal DRR threshold was 1.30 for both retroperitoneal lymph node involvement and metastasis. DRR was determined as an independent prognostic factor for retroperitoneal lymph node involvement and organ metastasis in univariate and multivariate analyses $(p<0.001, p=0.006$ and $\mathrm{p}=0.002, \mathrm{p}=0.047$, respectively).

Conclusions: A preoperative DRR greater than 1.30 may be an independent risk factor for retroperitoneal lymph node involvement and organ metastases in patients with TCa.

Keywords: Alanine transaminase; Aspartate aminotransferases; Neoplasm metastasis; Testicular neoplasms

This is an Open Access article distributed under the terms of the Creative Commons Attribution Non-Commercial License (http://creativecommons.org/licenses/by-nc/4.0) which permits unrestricted non-commercial use, distribution, and reproduction in any medium, provided the original work is properly cited.

\section{INTRODUCTION}

Testicular cancer (TCa) is responsible for $1 \%$ of solid organ neoplasms in men, and its incidence is increasing in Western countries [1-4]. Bilateral TCa is seen in $1 \%$ to $2 \%$ of cases at the time of diagnosis, and most of these cases are germ cell tumors [1].

The process of diagnosing TCa is begun and continued by determination of the level of tumor markers before and after surgical treatment, respectively. Follow-up

Received: 2 February, 2019 - Accepted: 3 March, 2019

Corresponding Author: Yigit Akin

Department of Urology, Izmir Katip Celebi University School of Medicine, 35620 Izmir, Turkey

TEL: +90-232-3293535, FAX: +90-232-3860888, E-mail: yigitakin@yahoo.com

ORCID: https://orcid.org/0000-0001-7627-3476

*These authors contributed equally to this study and should be considered co-first authors. 
regimens are also configured on the basis of these markers [5,6]. Alpha-fetoprotein (AFP) and human chorionic gonadotropin (hCG) are increased in almost $50 \%$ of patients with nonseminomatous germ cell tumors. Moreover, most nonseminomatous germ cell tumors present with an increase in one or both markers. Nearly one-third of seminomas can have increased hCG along the course of TCa [7]. Surgical treatment is the backbone of TCa treatment. Radical orchiectomy is one aspect of definitive treatment of TCa [8,9].

Amino transaminases, including aspartate aminotransferase (AST) and alanine aminotransferase (ALT), are enzymes produced in liver cells and released into the circulatory system. Because they can indicate hepatocellular damage, these enzymes are routinely included in the commonly requested enzyme panel used to evaluate liver function [10]. The ratio of serum activities of AST to ALT has been known as the De Ritis ratio (DRR) since it was first described by Fernando De Ritis [11]. This ratio (AST/ ALT) is also increased in liver diseases, specifically in viral hepatitis [12,13]. Published studies point to changing levels of amino transaminases [14]. Therefore, the DRR may be a prognostic biomarker in patients with malignant tumors [14]. In fact, these enzymes and the DRR have been reported as a significant prognostic biomarker in some malignancies [12-14]. However, the role of the DRR in TCa has not been presented in the published literature before.

In the present study, we evaluated the prognostic significance of preoperatively assessed DRR in the course of patients with TCa. Additionally, we described the threshold DRR for TCa metastasis.

\section{MATERIALS AND METHODS}

\section{Subjects and study design}

This was a retrospective evaluation of recorded data. All patients approved the study when they were diagnosed and provided signed consent forms. The Institutional Review Board of Izmir Katip Celebi University School of Medicine reviewed and approved the present study protocol (approval number: 2014/170). Patients with liver metastasis and/or viral hepatitis (HBV/HCV carrier), using liver-eliminated drugs, or with any liver disease (including any diseases that can cause liver fibrosis) were excluded. Clinical and pathology data for 128 patients with TCa who were admitted to our institution's urology outpatient clinic between March 2007 and January 2017 were assessed. Age; results of laboratory tests, including preoperative AFP, hCG, lactate dehydrogenase, AST, and ALT; tumor characteristics; clinical stage; pathological stage; pathological features of the tumor; tumor invasion to the spermatic cord; involvement into retroperitoneal lymph nodes; surgical margin; and any solid organ metastases were noted on Microsoft Excel data sheets. The primary outcomes were the presence of retroperitoneal lymph node involvement and metastasis.

\section{Diagnosis, definitions, and evaluation tools}

TCa was classified according to the 2009 TNM classification [15-17]. Preoperative retroperitoneal lymph node involvement and/or metastasis was evaluated with enhanced computed tomography. An automatic analyzer was used to determine AST and ALT levels by colorimetric methods. Upper levels of AST and ALT were $\geq 40 \mathrm{U} / \mathrm{L}$ and $\geq 35$ U/L, respectively. The levels of AST and ALT were assessed within 1 week of radical orchiectomy.

\section{Statistical analyses}

IBM SPSS Statistics ver. 22.0 (IBM Co., Armonk, NY, USA) was used to perform the statistical analyses. Receiver operating characteristic (ROC) curves were used to determine the potential prognostic value of the AST/ ALT ratio. The area under the ROC curve (AUC) with 95\% confidence interval (CI), sensitivity, and specificity are presented. DRR was recoded as a dichotomous variable according to its optimal cutoff to be tested in a multivariate logistic regression model. Odds ratios (ORs) were calculated for determining the testis tumor patients' prognosis according to the presence of retroperitoneal lymph node involvement and metastasis by univariate and multivariate logistic regression. Significance was defined as $\mathrm{p}<0.05$.

\section{RESULTS}

Mean follow-up was $37 \pm 9.7$ months. Five patients were excluded because of liver metastasis from TCa. Thus, 123 patients with TCa were enrolled in the present study. The patients' mean age was $39.3 \pm 10.3$ years. The minimum levels of AST and ALT were $19 \mathrm{U} / \mathrm{L}$ and $8 \mathrm{U} / \mathrm{L}$, respectively. The maximum levels of AST and ALT were $71 \mathrm{U} / \mathrm{L}$ and $57 \mathrm{U} / \mathrm{L}$, respectively. Demographic and clinical data are summarized in Table 1 . There were 45 and 73 TCa patients with any metastasis and without lymph node involvement, respectively. For both presence of retroperitoneal lymph node involvement and metastasis, an AST/ALT ratio of 1.30 was determined as an optimal threshold (Fig. 1, AUC: 0.674; Fig. 2, AUC: 0.705). Fig. 1 shows 45 metastatic TCa patients. Thus, a variety of tests were used to evaluate the risk of any metastasis.

Univariate analyses were done to evaluate the presence 
Table 1. Demographic and clinical data of patients with testicular cancer $(n=123)$

\begin{tabular}{|c|c|}
\hline Variable & Value \\
\hline Age (y) & $39.32 \pm 10.36$ \\
\hline Preoperative AST (U/L) & $20(19-71)$ \\
\hline Preoperative ALT (U/L) & $20(8-57)$ \\
\hline Preoperative AST/ALT & $1.20 \pm 0.50$ \\
\hline Preoperative AFP (ng/mL) & $5(1-54,260)$ \\
\hline Preoperative hCG (mU/mL) & $2(0-1,164,157)$ \\
\hline Preoperative LDH (U/L) & $221(114-2,782)$ \\
\hline \multicolumn{2}{|l|}{ Lymphovascular invasion } \\
\hline Negative & $78(63.4)$ \\
\hline Positive & $45(36.6)$ \\
\hline \multicolumn{2}{|l|}{ Pathological T stage } \\
\hline T0 & $0(0.0)$ \\
\hline $\mathrm{T} 1$ & $78(63.4)$ \\
\hline $\mathrm{T} 2$ & $33(26.8)$ \\
\hline T3 & $12(9.8)$ \\
\hline T4 & $0(0.0)$ \\
\hline \multicolumn{2}{|l|}{ Epididymis invasion } \\
\hline Negative & $113(91.9)$ \\
\hline Positive & $10(8.1)$ \\
\hline \multicolumn{2}{|l|}{ Spermatic cord invasion } \\
\hline Negative & $111(90.2)$ \\
\hline Positive & $12(9.8)$ \\
\hline \multicolumn{2}{|l|}{ Surgical margin } \\
\hline Negative & $120(97.6)$ \\
\hline Positive & $3(2.4)$ \\
\hline \multicolumn{2}{|c|}{ Retroperitoneal lymph node involvement } \\
\hline Negative & $78(63.4)$ \\
\hline Positive & $45(36.6)$ \\
\hline \multicolumn{2}{|l|}{ Lung metastasis } \\
\hline Negative & $109(88.6)$ \\
\hline Positive & $14(35.2)$ \\
\hline \multicolumn{2}{|l|}{ Extrapulmonary metastasis } \\
\hline Negative & $119(96.7)$ \\
\hline Positive & $4(3.3)$ \\
\hline
\end{tabular}

Values are presented as mean \pm standard deviation, median (minmax), or number (\%).

AST, aspartate aminotransferase; ALT, alanine aminotransferase; AFP, alpha fetoprotein; hCG, beta human chorionic gonadotropin; LDH, lactate dehydrogenase.

of retroperitoneal lymph node involvement and rete testis involvement. These analyses suggested 1.30 for DRR as the threshold for lymph node involvement and/or metastases. In the multivariate logistic regression model, age had a protective effect (OR, 0.946; 95\% CI, 0.907-0.986), although a preoperative hCG level of 5,000 $\mathrm{mU} / \mathrm{mL}$ and above and a DRR of 1.30 and above were risk factors (OR, 6.170; 95\% CI, 1.122-33.934; OR, 3.830; 95\% CI, 1.478-9.924) in the assessment of metastasis.

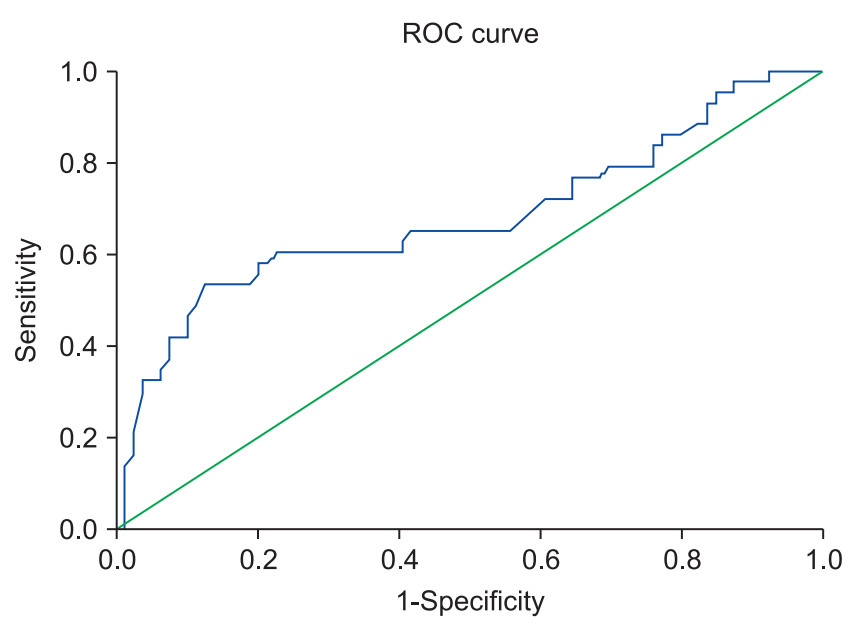

Diagonal segments are produced by ties.

Fig. 1. The risk of retroperitoneal lymph node involvement in testicular cancer patients at a De Ritis ratio level of 1.30 (area under the curve, $0.674 ; 95 \%$ confidence interval, $0.563-0.786$ ). ROC curve, receiver operating characteristic curve.

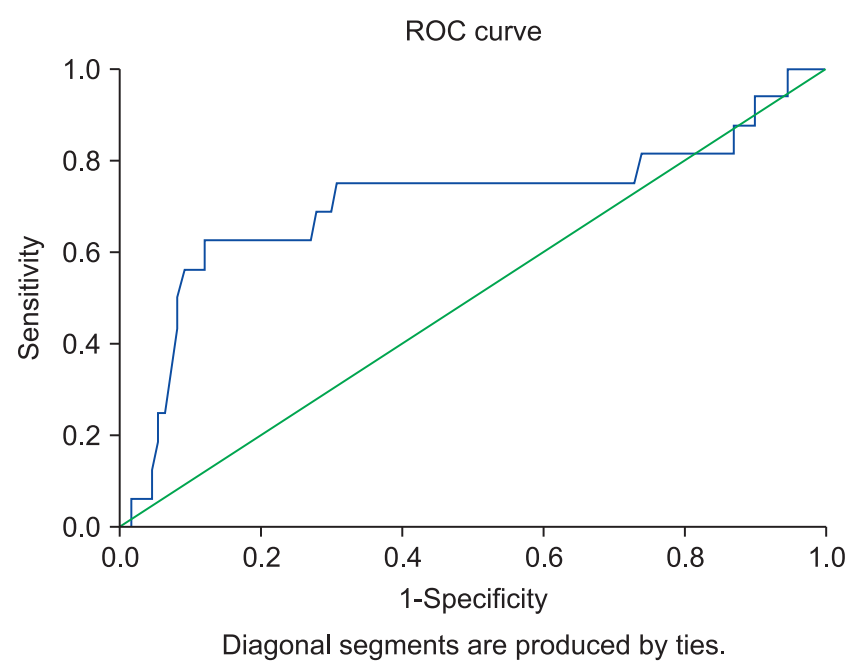

Fig. 2. The risk of metastasis in testicular cancer patients at a De Ritis ratio level of 1.30 (area under the curve, $0.705 ; 95 \%$ confidence interval, 0.535-0.875). ROC curve, receiver operating characteristic curve.

Tumor size, preoperative hCG, lymphovascular invasion, rete testis involvement, and a DRR of 1.30 and above were risk factors in the univariate analyses for metastasis (OR, 1.222; 95\% CI, 1.039-1.438; OR, 12.240; 95\% CI, 3.164-47.352; OR, 3.429; 95\% CI, 1.153-10.195; OR, 8.571; 95\% CI, 2.408-30.506; OR, 6.441; 95\% CI, 1.935-21.440). In the multivariate regression model, only a preoperative hCG level of 5,000 mU/mL and above and a DRR of 1.30 and above were risk factors (OR, 16.253; 95\% CI, 2.783-94.906; OR, 4.561; 95\% CI, 1.021-20.375) (Tables 2, 3). We also showed survival in Kaplan-Meier curves for retroperitoneal lymph node involvement (Fig. 3A) and metastasis (Fig. 3B). 
Table 2. ORs on univariate analyses for presence of retroperitoneal lymph node involvement and metastasis in testis tumor patients

\begin{tabular}{|c|c|c|c|c|}
\hline \multirow[t]{2}{*}{ Parameter } & \multicolumn{2}{|c|}{$\begin{array}{l}\text { Retroperitoneal lymph node involvement } \\
\text { (univariate) }\end{array}$} & \multicolumn{2}{|c|}{$\begin{array}{l}\text { Metastasis } \\
\text { (univariate) }\end{array}$} \\
\hline & OR $(95 \% \mathrm{Cl})$ & p-value & OR $(95 \% \mathrm{Cl})$ & p-value \\
\hline Age & $0.946(0.907-0.986)$ & 0.212 & $1.006(0.957-1.058)$ & 0.817 \\
\hline Tumor size & $1.081(0.952-1.227)$ & 0.231 & $1.222(1.039-1.438)$ & $0.015^{*}$ \\
\hline Preoperative hCG ( $<5,000$ vs. $\geq 5,000)$ & $10.191(2.090-49.698)$ & $0.004^{*}$ & $12.240(3.164-47.352)$ & $<0.001^{*}$ \\
\hline Lymphovascular invasion (negative \& positive) & $1.993(0.925-4.293)$ & 0.078 & $3.429(1.153-10.195)$ & $0.027^{*}$ \\
\hline Rete testis involvement (negative \& positive) & $4.286(1.209-15.192)$ & $0.024^{*}$ & $8.571(2.408-30.506)$ & $0.001^{*}$ \\
\hline De Ritis ratio (AST/ALT, $<1.30 \& \geq 1.30$ ) & $4.830(2.170-10.748)$ & $<0.001^{*}$ & $6.441(1.935-21.440)$ & $0.002^{*}$ \\
\hline ALT & $0.953(0.912-0.995)$ & $0.028^{*}$ & $0.968(0.912-1.027)$ & 0.282 \\
\hline AST & $1.005(0.954-1.059)$ & 0.842 & $1.013(0.945-1.087)$ & 0.712 \\
\hline
\end{tabular}

$\mathrm{OR}$, odds ratio; $\mathrm{Cl}$, confidence interval; hCG, beta human chorionic gonadotropin; AST, aspartate aminotransferase; ALT, alanine aminotransferase.

*Statistically significant, $\mathrm{p}<0.05$.

Table 3. Adjusted OR and 95\% Cls for clinical outcomes in testis tumor patients in multivariate analyses

\begin{tabular}{|c|c|c|c|c|}
\hline \multirow{2}{*}{ Parameter } & \multicolumn{2}{|c|}{ Retroperitoneal lymph node involvement } & \multicolumn{2}{|c|}{ Metastasis } \\
\hline & OR $(95 \% \mathrm{Cl})$ & p-value & OR $(95 \% \mathrm{Cl})$ & p-value \\
\hline De Ritis ratio (<1.30 vs. $\geq 1.30)$ & $3.830(1.478-9.924)$ & $<0.006^{*}$ & $4.561(1.021-20.375)$ & $0.047^{*}$ \\
\hline Preoperative hCG (<5,000 vs. $\geq 5,000)$ & $6.170(1.122-33.934)$ & $0.036^{*}$ & $16.253(2.783-94.906)$ & $0.002^{*}$ \\
\hline
\end{tabular}

$\mathrm{OR}$, odds ratio; $\mathrm{Cl}$, confidence interval; $\mathrm{hCG}$, beta human chorionic gonadotropin.

*Statistically significant, $p<0.05$.
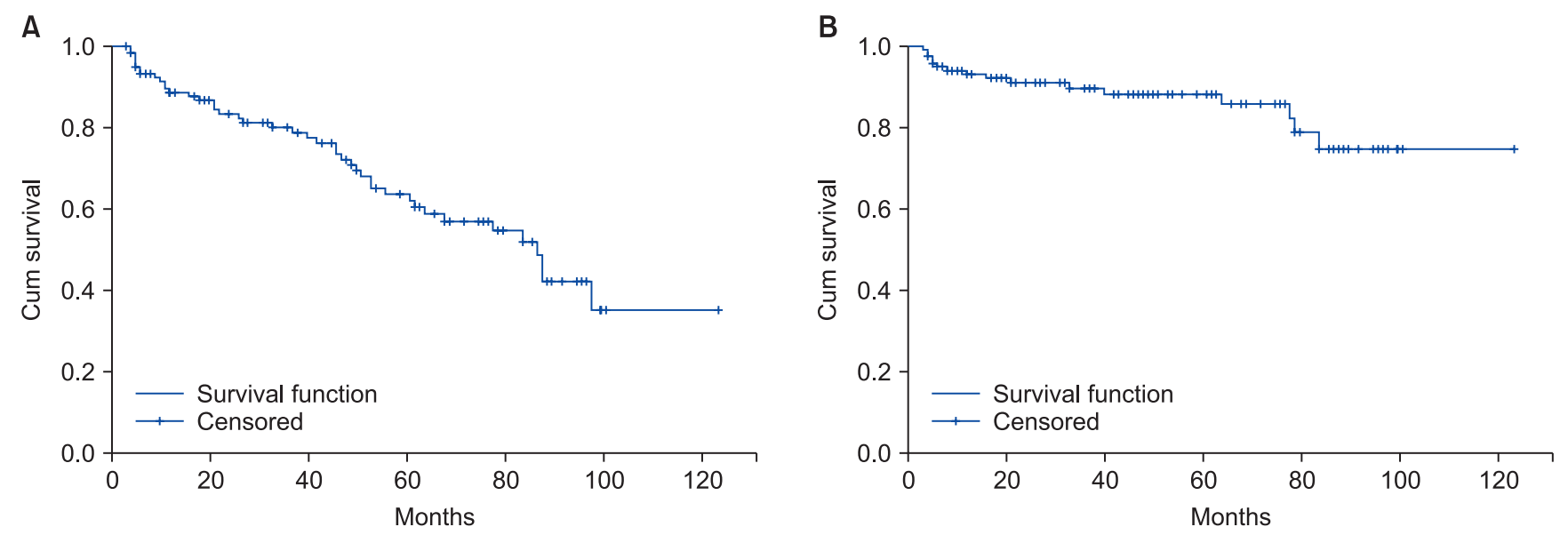

Fig. 3. Survival functions in Kaplan-Meier curves. (A) Retroperitoneal lymph node involvement. (B) Metastasis.

\section{DISCUSSION}

We investigated the relationship between the DRR and prognosis of TCa. Although we had a small patient population, we recorded 45 patients with TCa (36.6\%) who had metastases. According to these findings, we determined the threshold DRR to be 130. Thus, our findings suggest that a preoperative DRR higher than 1.30 may be a sign of organ metastases or retroperitoneal lymph node involvement.

Diagnosis of TCa depends on clinical, laboratory, and radiological features and tumor markers [18]. From another point of view, however, none of these can accurately pre- dict with certainty the presence of future metastases. If retroperitoneal lymph node involvement or metastasis is not detected in radiologic examinations, markers cannot provide information for metastases or retroperitoneal lymph node involvement. Here we showed evidence of the opposite: that an increased preoperative DRR might be an indicator for retroperitoneal lymph node involvement or organ metastases in TCa. In this way, the DRR can provide clinicians a means of predicting retroperitoneal lymph node involvement and metastases before inguinal orchiectomy.

Serum tumor markers are important in preoperative and postoperative follow-up for TCa. It is well known that 
the mean serum half-lives of $\mathrm{hCG}$ and AFP are 3 days and 5 days, respectively [7]. Thus, TCa tumor markers should be reevaluated after surgery, taking into account the half-life of tumor markers. In view of this, patients with clinical stage $1 \mathrm{TCa}$ should undergo reassessment until normal values are measured. Markers are essential for categorizing TCa patients before starting chemotherapy [19]. The detection of elevated serum tumor markers after orchiectomy may be a sign of metastasis (macro- or micro). On the other hand, clinicians should be cognizant that normal values of a marker after orchiectomy do not exclude tumor metastases. Moreover, tumor markers should be followed-up, and persistence of elevated marker levels indicates a poor prognosis during chemotherapy [20,21].

AST is produced in different tissues, whereas ALT is regarded as liver-specific [22]. In addition, some conditions like pathologic diseases with high proliferative properties, organ damage, and tumors with high cell turnover can elevate AST rather than ALT [22]. All these variations in levels of ALT and AST make DRR a candidate as a possible biomarker. The use of DRR as a biomarker has been reported previously in the published literature. Tan et al. concluded that a DRR of more than 2.0 might be a useful biomarker for determining survival in distal cholangiocarcinoma [21-23]. Dorward et al. [24] reported abnormal anaerobic glycolysis in cancer cells to produce adenosine triphosphate (ATP). Rapid tumor growth is related to increased ATP production, and this is used to determine mechanisms in tumor-specific imaging and antitumor therapy [25]. Increased glycolysis is also related to mitochondrial activities involved with nicotinamide adenine dinucleotide enzymes and glucose transporters [26]. Because tumor cells produce ATP faster, they prefer anaerobic glycolysis to oxidative phosphorylation [27]. In view of these data, an elevated DRR can be used as one indicator of tumor activity. In the literature, only a few studies have shown a connection between DRR and urologic cancers [28-30]. In these studies, the increased DRR was associated with poor prognosis. However, no published study has shown a relationship between DRR and TCa. In the present paper, we showed that an elevated DRR may predict possible metastasis (such as lymph node involvement and metastasis) of TCa. Either our results are parallel to the literature or this is the first study giving eligible proof of metastases in TCa patients with elevated DRR. Additionally, this is related to poor prognostic factors.

Although the current study reported new findings, it had several limitations. First, the study was performed in a retrospective manner and had a small patient population. Second, the DRR can be changed by undetected liver diseases, other diseases, and alcohol consumption, which can affect the levels of ALT and AST. This may suggest a large selection bias. However, this is the first study in the literature on this issue. Additionally, we did not compare perioperative and postoperative DRR. High levels of DRR were related to the presence of TCa. Surgical removal of the tumor, of course, leads to decreased DRR. Third, drug interactions could affect liver functions, leading to increased AST and ALT. Besides, retroperitoneal lymph node involvement and metastases were evaluated in computed tomography scans. Additionally, these were compared after treatments during follow-up. This might suggest a confounding bias. Moreover, we did not perform any molecular study. Furthermore, our results can be considered a pathfinder study in the way of testis-sparing surgery. However, testis-sparing surgery is still under debate. More series with higher patient volume are needed.

This is the first study in the literature that investigated the impact of amino transaminase on the prognosis of patients with TCa before surgery. In addition, AST and ALT are used in clinicians' daily practice. We identified that an increased DRR may be related to worsening prognosis in patients with TCa. Twelve patients died because of TCa; for this reason, we could not perform any survival analysis. Survival analysis should be performed for a larger series of patients in future studies.

\section{CONCLUSIONS}

An elevated DRR may be an independent risk factor for retroperitoneal lymph node involvement or metastases in patients with $\mathrm{TCa}$ at the time diagnosis, before surgery. If the DRR is higher than 130, clinicians should reevaluate to detect possible retroperitoneal lymph node involvement and metastases in TCa. Therefore, clinicians should inform patients before the operation concerning metastases in the case of a DRR <1.30, independent of radiological examinations. Our results need to be confirmed with prospective studies including larger sample sizes, including molecular studies.

\section{CONFLICTS OF INTEREST}

The authors have nothing to disclose.

\section{ACKNOWLEDGMENTS}

This study was presented at the Euroasian Urooncology Congress, June 28-July 1, 2018, in Tblisi, Georgia. 


\section{REFERENCES}

1. La Vecchia C, Bosetti C, Lucchini F, Bertuccio P, Negri E, Boyle P, et al. Cancer mortality in Europe, 2000-2004, and an overview of trends since 1975. Ann Oncol 2010;21:1323-60.

2. Osbun N, Winters B, Holt SK, Schade GR, Lin DW, Wright JL. Characteristics of patients with sertoli and leydig cell testis neoplasms from a national population-based registry. Clin Genitourin Cancer 2017;15:e263-6.

3. Jemal A, Siegel R, Ward E, Hao Y, Xu J, Thun MJ. Cancer statistics, 2009. CA Cancer J Clin 2009;59:225-49.

4. Nigam M, Aschebrook-Kilfoy B, Shikanov S, Eggener S. Increasing incidence of testicular cancer in the United States and Europe between 1992 and 2009. World J Urol 2015;33:623-31.

5. Germà-Lluch JR, Garcia del Muro X, Maroto P, Paz-Ares L, Arranz JA, Gumà J, et al.; Spanish Germ-Cell Cancer Group (GG). Clinical pattern and therapeutic results achieved in 1490 patients with germ-cell tumours of the testis: the experience of the Spanish Germ-Cell Cancer Group (GG). Eur Urol 2002;42:553-62.

6. Wanderås EH, Tretli S, Fosså SD. Trends in incidence of testicular cancer in Norway 1955-1992. Eur J Cancer 1995;31A: 2044-8.

7. Peyret C. Tumeurs du testicule. Synthèse et recommandations en onco-urologie [Testicular tumours. Summary of oncourological recommendations]. Prog Urol 1993;2:60-4. French.

8. Koshida K, Uchibayashi T, Yamamoto H, Hirano K. Significance of placental alkaline phosphatase (PLAP) in the monitoring of patients with seminoma. Br J Urol 1996;77:138-42.

9. Mottet N, Rousmans S, Culine S. [Systematic review 2007: primary treatments of testicular germ cell tumors after radical orchydectomy]. Bull Cancer 2008;95:205-34. French.

10. Ozer J, Ratner M, Shaw M, Bailey W, Schomaker S. The current state of serum biomarkers of hepatotoxicity. Toxicology 2008;245:194-205.

11. De Ritis F, Coltorti M, Giusti G. An enzymic test for the diagnosis of viral hepatitis; the transaminase serum activities. Clin Chim Acta 1957;2:70-4.

12. Botros M, Sikaris KA. The de ritis ratio: the test of time. Clin Biochem Rev 2013;34:117-30.

13. Rawson NS, Peto J. An overview of prognostic factors in small cell lung cancer. A report from the Subcommittee for the Management of Lung Cancer of the United Kingdom Coordinating Committee on Cancer Research. Br J Cancer 1990;61:597-604.

14. Stocken DD, Hassan AB, Altman DG, Billingham LJ, Bramhall SR, Johnson PJ, et al. Modelling prognostic factors in advanced pancreatic cancer. Br J Cancer 2008;99:883-93.

15. Sobin LH, Gospodarowicz MK, Wittekind C. TNM classification of malignant tumors. In: Sobin LH, Gospodarowicz MK,
Wittekind C (ed.) UICC International Union Against Cancer. 7th ed. Oxford: Wiley-Blackwell; 2009;249-54.

16. Secil M, Altay C, Basara I. State of the art in germ cell tumor imaging. Urol Oncol 2016;34:156-64.

17. Masterson TA, Shayegan B, Carver BS, Bajorin DF, Feldman DR, Motzer RJ, et al. Clinical impact of residual extraretroperitoneal masses in patients with advanced nonseminomatous germ cell testicular cancer. Urology 2012;79:156-9.

18. Haugnes HS, Laurell A, Stierner U, Bremnes RM, Dahl O, Cavallin-Ståhl E, et al. High-dose chemotherapy with autologous stem cell support in patients with metastatic nonseminomatous testicular cancer - a report from the Swedish Norwegian Testicular Cancer Group (SWENOTECA). Acta Oncol 2012;51:168-76.

19. Fizazi K, Culine S, Kramar A, Amato RJ, Bouzy J, Chen I, et al. Early predicted time to normalization of tumor markers predicts outcome in poor-prognosis nonseminomatous germ cell tumors. J Clin Oncol 2004;22:3868-76.

20. Panchuk RR, Skorokhyd NR, Kozak YS, Lehka LV, Chumak VV, Omelyanchik SN, et al. Antioxidants selenomethionine and D-pantethine decrease the negative side effects of doxorubicin in NL/Ly lymphoma-bearing mice. Croat Med J 2016;57:180-92.

21. Tan X, Xiao K, Liu W, Chang S, Zhang T, Tang H. Prognostic factors of distal cholangiocarcinoma after curative surgery: a series of 84 cases. Hepatogastroenterology 2013;60:1892-5.

22. Warburg O. On respiratory impairment in cancer cells. Science 1956;124:269-70.

23. Walker-Samuel S, Ramasawmy R, Torrealdea F, Rega M, Rajkumar V, Johnson SP, et al. In vivo imaging of glucose uptake and metabolism in tumors. Nat Med 2013;19:1067-72.

24. Dorward A, Sweet S, Moorehead R, Singh G. Mitochondrial contributions to cancer cell physiology: redox balance, cell cycle, and drug resistance. J Bioenerg Biomembr 1997;29:38592.

25. Fantin VR, St-Pierre J, Leder P. Attenuation of LDH-A expression uncovers a link between glycolysis, mitochondrial physiology, and tumor maintenance. Cancer Cell 2006;9:425-34.

26. Bezan A, Mrsic E, Krieger D, Stojakovic T, Pummer K, Zigeuner $\mathrm{R}$, et al. The preoperative AST/ALT (De Ritis) ratio represents a poor prognostic factor in a cohort of patients with nonmetastatic renal cell carcinoma. J Urol 2015;194:30-5.

27. Wang H, Fang K, Zhang J, Jiang Y, Wang G, Zhang H, et al. The significance of De Ritis (aspartate transaminase/alanine transaminase) ratio in predicting pathological outcomes and prognosis in localized prostate cancer patients. Int Urol Nephrol 2017;49:1391-8.

28. Gorgel SN, Kose O, Koc EM, Ates E, Akin Y, Yilmaz Y. The prognostic significance of preoperatively assessed AST/ALT 
(De Ritis) ratio on survival in patients underwent radical cystectomy. Int Urol Nephrol 2017;49:1577-83.

29. Ha YS, Kim SW, Chun SY, Chung JW, Choi SH, Lee JN, et al. Association between De Ritis ratio (aspartate aminotransferase/alanine aminotransferase) and oncological outcomes in bladder cancer patients after radical cystectomy. BMC Urol
2019;19:10.

30. Nishikawa M, Miyake H, Fujisawa M. De Ritis (aspartate transaminase/alanine transaminase) ratio as a significant predictor of recurrence-free survival in patients with upper urinary tract urothelial carcinoma following nephroureterectomy. Urol Oncol 2016;34:417.e9-15. 